\title{
High-z radio-loud QSOs observed with INTEGRAL
}

\author{
A. De Rosa*, S. Gianní, P. Ubertini \\ INAF/IASF-Roma, via del Fosso del cavaliere 100, I-00133 Roma, Italy \\ E-mail: alessandra.derosa@iasf-roma.inaf.it,
}

\section{Bassani, A. Malizia}

INAF/IASF-Bologna, via Gobetti 101, I-40129 Bologna, Italy

\section{A. J. Dean}

School of Physics and Astronomy, University of Southampton Highfield, Southampton, United Kingdom

\begin{abstract}
We present the study of a sample 5 of "high-z" radio-loud QSOs (from $\mathrm{z}=0.1$ to $\mathrm{z}=3.668$ ) observed with INTEGRAL and XMM-Newton. The broadband coverage $(0.2-100 \mathrm{keV})$ allowed us to investigate in depth the intrinsic continuum together with the absorption/emission spectral features in the soft X-ray energy range. All but one (4C04.42) sources show evidence of spectral flattening below 2-3 keV (observer frame). In the past this behaviour has been attributed either to cold/ionized absorption at the redshift of the source or to intrinsic break of the continuum. We report here, for our sample, the distribution of the absorption column density $\mathrm{N}_{\mathrm{H}}$ and that of the intrinsic photon index. This study shows evidence that the hard-X ray selection favours "flat" sources and is able to unveils heavily absorbed objects. 4C04.42 and IGR J22517+2218 are peculiar sources among the sample: 4C04.42 shows possible evidence of bulk Compton component in the soft X-ray energy range, while IGR J22517+2218 is the farthermost QSO detected so far by INTEGRAL and represents a shining lighthouse at the edge of our Universe. For both objects a detailed spectral analysis is presented and the not-simultaneous Spectral Energy Distribution of the sources from radio to gamma ray frequencies is studied in detail.
\end{abstract}

7th INTEGRAL Workshop

September 8-11 2008

Copenhagen, Denmark

\footnotetext{
* Speaker.
} 


\section{Introduction}

Blazars are the most powerful objects in the observable Universe, they emit from radio frequencies up to the extreme gamma-rays. In the X-ray energy range, harder spectra are associated with the highest luminosity objects [13], and Flat Spectrum Radio Quasars (FSRQ) are the most luminous class of Blazars. The Spectral Energy Distribution (SED) of FSRQ exhibits two main peaks (one between the IR and soft X-rays and the other in the gamma-rays), disclosing the presence of two main components: it is widely believed that the low energy one is due to the Synchrotron radiation of relativistic electrons in a jet, while the high energy one is due to Inverse Compton scattering (IC) of the same electrons with a photon field [14]. It is also believed that in FSRQ the IC is due to Compton scattering of photons external to the jet (external Compton radiation EC), probably produced in the accretion disk and reprocessed by the Broad Line Region (BLR) and/or the dusty torus $[7,28]$. Other competitive processes, like the IC from photons produced by Synchrotron (Synchrotron Self-Compton radiation, SSC) can contribute to the high energy component [18]. The investigation of these extreme objects in the X-ray band is important mainly because they emit most of their bolometric luminosity in this energy range [8]. Observations have demonstrated that radio-loud QSOs are more powerful X-ray emitters than radio-quiet counterparts or RQQs [22] and, therefore, more easily observable. Various authors [24, 23] have demonstrated that radio-loud QSOs exhibit a flatter intrinsic spectral shape $(\Gamma \sim 1.6)$ than RQQs $(\Gamma \sim 1.9)$; this harder X-ray emission is thought to be Synchrotron or IC emission from the relativistic radio jet, rather than from the accretion disk.

A deficit of photons in the soft X-ray band ( $\mathrm{E}<2 \mathrm{keV}$ observer frame) of several high and low-z QSOs has been observed in the past by ASCA [9, 11], and recently confirmed through XMMNewton, Chandra and Swift observations [1,27, 26]. The origin of this feature is not yet clear. Possible hypotheses are an intrinsic cold/ionized absorption or an intrinsic break of the continuum. When associated with absorption a clear trend of $\mathrm{N}_{\mathrm{H}}$ vs $\mathrm{z}$ has been measured, indicating a cosmic evolution effect, which seems to be strongest at redshifts around 2 [27]. However, excess emission at similar low energies has also been observed, but only in a few sources [16, 25]. The origin of this behaviour is still unclear, even if several scenarios have been proposed and explored, like Bulk Comptonization [3], increasing contribution of the SSC component, [16], and reflection from the accretion disk [25]. So far these two spectral features (excess or deficit of soft X-ray photons), have been interpreted as due to different emission processes. However, very recently, [3] modeled the soft X-ray flattening observed in the quasar BG B1428-4217, via bulk Comptonization process. Clearly more sources need to be observed in order to improve the statistics.

Here we present a broadband analysis of a sample of 5 QSOs detected by INTEGRAL above $10 \mathrm{keV}$. The data below $10 \mathrm{keV}$ are collected through XMM-Newton and Swift observations. The sources are the highest-z objects belonging to the 3rd IBIS/ISGRI catalogue [2]. The general properties of the sample and a spectral analysis of each single source are presented. Among the QSOs in the sample 4C04.42 and IGR J22517+2218 are peculiar: 4C04.42 [5] shows possible evidence of bulk Compton motion, while IGR J22517+2218 [1] is the most distant object observed so far by INTEGRAL, hence a source with a huge amount of luminosity emitted in hard X-rays. These objects are discussed here in detail ${ }^{1}$.

\footnotetext{
${ }^{1}$ Throughout this paper we adopt a $\Lambda \mathrm{CDM}$ cosmology with $\Omega_{m}=0.27, \Omega_{\Lambda}=0.73$ and $\mathrm{H}_{0}=71 \mathrm{~km} \mathrm{~s}^{-1} \mathrm{Mpc}^{-1}$.
} 
Table 1: High-z radio-loud QSOs in the 3rd INTEGRAL catalogue

\begin{tabular}{ccccccc}
\hline Name & R.A. & Dec. & $\mathrm{z}$ & ${ }^{1} \mathrm{~N}_{\mathrm{H}}^{\text {gal }}$ & ${ }^{2} \mathrm{t}_{\exp }$ & ref \\
\hline PKS 1830-211 & 103339.89 & -210339.8 & 2.507 & 0.26 & 2000 & {$[6,12]$} \\
Swift J1656.3-3302 & 165616.56 & 330209.3 & 2.4 & 0.22 & 2000 & {$[20]$} \\
IGR J22517+2218 & 225153.50 & +221737.3 & 3.668 & 0.05 & 262.5 & {$[1,17]$} \\
QSO B0836+710 & 084124.37 & +705342.2 & 2.172 & 0.03 & 768 & - \\
4C 04.42 & 122222.55 & +041315.8 & 0.965 & 0.017 & 1798 & {$[5]$} \\
\hline
\end{tabular}

${ }^{1}$ Galactic hydrogen column density in units of $10^{22} \mathrm{~cm}^{-2} \cdot{ }^{2}$ Exposure time INTEGRAL/IBIS in units of ks.

\section{High-z QSOs in the 3rd INTEGRAL survey}

We describe here the broadband $(0.2-100 \mathrm{keV})$ analysis of a sample of 5 objects that are representative of the "high z" QSOs belonging all but one (IGR J22517+2218), to the third IBIS/ISGRI $\mathrm{X}$-ray Survey Catalogue [2]. The data below $10 \mathrm{keV}$ have been collected through XMM-Newton and Swift observations. These objects, as well as the log of the available observations, are listed in Table 1 . The observed flux in the sample ranges between $(0.8-2) \times 10^{-11} \mathrm{erg} \mathrm{cm}^{-2} \mathrm{~s}^{-1}(1-3 \mathrm{mCrab})$ in $20-40 \mathrm{keV}$, and between $(1-4) \times 10^{-11} \mathrm{erg} \mathrm{cm}^{-2} \mathrm{~s}^{-1}(1-4 \mathrm{mCrab})$ in $40-100 \mathrm{keV}$. As far as it concerns their distance, the selected objects cover a redshift range of $0.965 \leq z \leq 3.668$. All but one (4C04.42) sources were well fitted, in $0.2-100 \mathrm{keV}$ energy range, with an absorbed power-law model. Results of this fit are shown in Table 2, for all sources galactic absorption is taken into account. The distribution of the hydrogen column density (rest frame) of the cold absorber as obtained from the broadband analysis, is shown in the left panel in Figure 1. The distribution of $\mathrm{N}_{\mathrm{H}}$ seems to be asymmetric, with a peak located at about $\log \mathrm{N}_{\mathrm{H}}=23$, and a tail extending until $\log$ $\mathrm{N}_{\mathrm{H}}=20$. It is worth noting that the statistical significance of this result is strongly affected by the small number of objects in our sample.

In order to perform a direct comparison of our results with those obtained for a larger sample of QSOs observed in 0.3-10 keV with XMM-Newton [22], we reanalyzed the sample in the spectral range $0.2-10 \mathrm{keV}$. In the right panel in Figure 2 we show the comparison between the distributions of $N_{H}$ for the two samples. A quick look to this figure reveals that the location of the peaks of the two distributions are in good agreement $\left(\log \mathrm{N}_{\mathrm{H}}^{\text {peak }}=22\right)$. Looking at the distributions obtained through a 2-10 keV energy band data analysis, we note that sources with absorbing column density $\log \mathrm{N}_{\mathrm{H}} \geq 23$ are missing. This evidence is expected in view of the fact that in the soft X-ray energy range these sources are almost completely obscured. On the other hand, by comparing the distributions of $\mathrm{N}_{\mathrm{H}}$ as obtained through spectral analysis in $0.2-100 \mathrm{keV}$ and $0.2-10 \mathrm{keV}$, we note that a tail towards the high values of column densities appears. This strongly suggests that observations above $10 \mathrm{keV}$ represent a strong tool to make an unbiased selection of heavily absorbed objects. In Figure 2 we show the distribution of the photon indices $(\Gamma)$ for all the sources so far investigated, as obtained by performing the fit in the spectral range $0.2-100 \mathrm{keV}$. The mean value is $\Gamma=1.3 \pm 0.1$. This value results to be flatter than that found by Page et al. (2005) analyzing a sample of radio-loud objects in $0.3-10 \mathrm{keV}$ energy band $(\Gamma=1.55 \pm 0.04)$. The difference could be due to the hard X-ray selection of our INTEGRAL sample, that is clearly biased towards the 
Table 2: High-z INTEGRAL QSOs: best fit model parameters

\begin{tabular}{ccccccc}
\hline \multicolumn{7}{c}{ XMM + INTEGRAL } \\
\hline Name & ${ }^{1} \mathrm{~F}_{o b s}^{2-10 k e V}$ & ${ }^{1} \mathrm{~F}_{o b s}^{20-100 k \mathrm{kV}}$ & ${ }^{2} \mathrm{C}$ & ${ }^{3} \mathrm{~N}_{H}$ & $\Gamma$ & $\chi_{v}^{2} / \mathrm{dof}$ \\
\hline QSO B0836+710 & 4.0 & 17.7 & $0.28 \pm 0.03$ & $0.07 \pm 0.02$ & $1.358 \pm 0.005$ & $1.026 / 2423$ \\
PKS 1830-211 & 1.5 & 12.2 & $0.36 \pm 0.03$ & $1.80 \pm 0.10$ & $1.11 \pm 0.02$ & $1.11 / 1013$ \\
\hline \multicolumn{7}{c}{ Swift/XRT + INTEGRAL } \\
\hline Swift J1656.3-3302 & 0.5 & 2.0 & $1.9_{-0.9}^{+1.7}$ & $7.9_{-3.8}^{+5.1}$ & $1.66_{-0.20}^{+0.22}$ & $1.027 / 42$ \\
IGR J22517+2218 & 0.2 & 3.5 & $7.1_{-3.4}^{+6.7}$ & $3.6_{-2.2}^{+3.3}$ & $1.66_{-0.19}^{+0.21}$ & $0.845 / 49$ \\
\hline
\end{tabular}

${ }^{1}$ Observed flux in units of $10^{-11} \mathrm{erg} \mathrm{cm}^{-2} \mathrm{~s}^{-1}$. ${ }^{2}$ IBIS/EPIC-MOS and IBIS/XRT cross calibration constant.

${ }^{3}$ Hydrogen column density in units of $10^{22} \mathrm{~cm}^{-2}$.
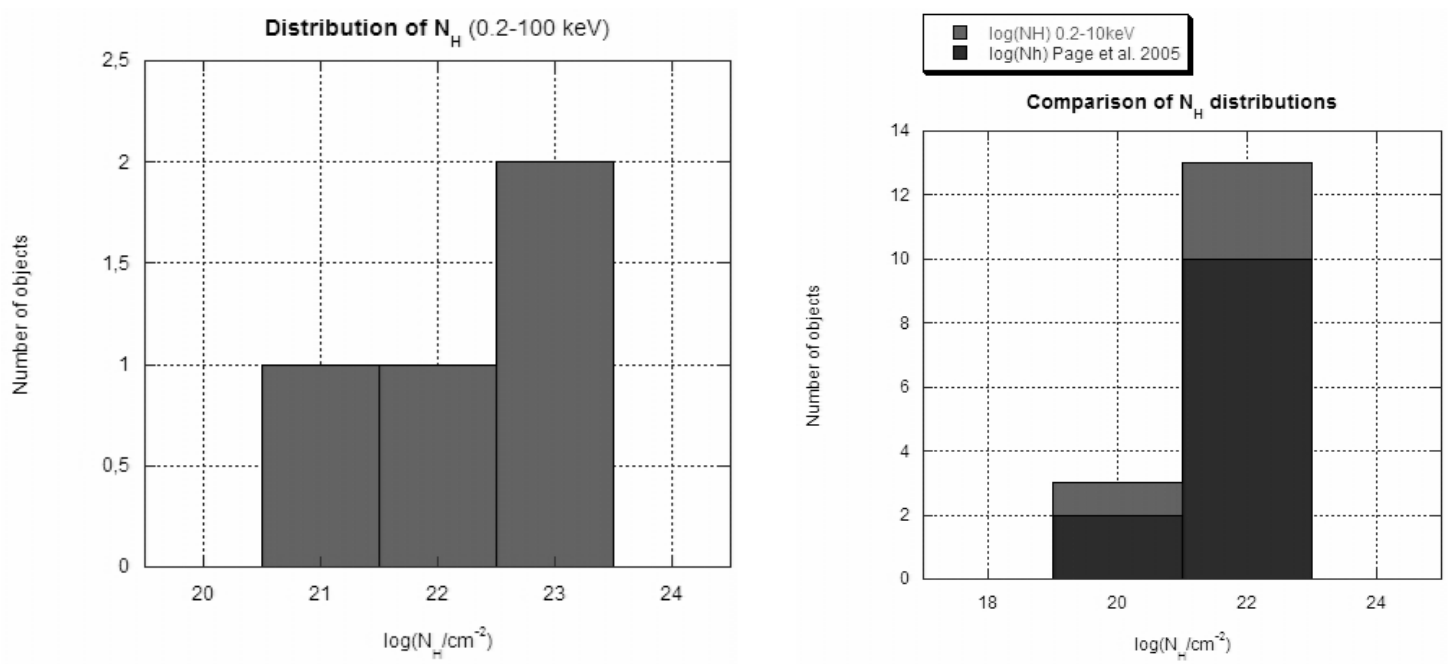

Figure 1: Distributions of the hydrogen column densities for the absorber associated with the quasars (at the quasar redshifts) in the sample. In the left panel are shown the results obtained through a broadband analysis $(0.2-100 \mathrm{keV})$, while in the right panel a comparison between the distribution obtained for our sample analyzed in 0.2-10 keV and that obtained for a larger sample of QSOs observed with XMM-Newton[22].

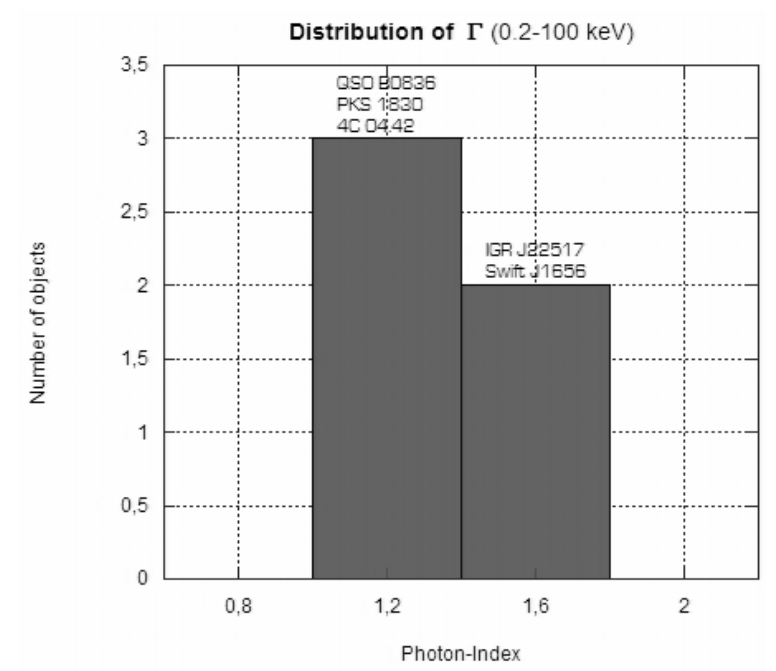

Figure 2: Distribution of the photon indices in the sample. 


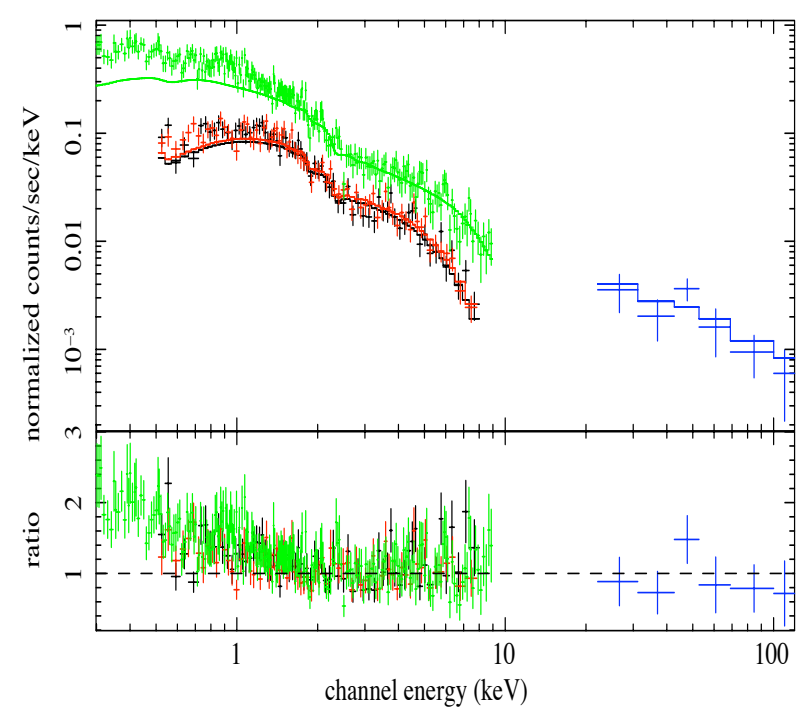

Figure 3: 4C04.42. Continuum power-law fit to the energy band above $2 \mathrm{keV}$ (observer frame) extrapolated over the low energy range. Green curve: pn data, black and red curves: MOS1, MOS2 data.

flatter values of the photon index.

\section{The FSRQ 4C04.42}

4C04.42 was detected above $20 \mathrm{keV}$ by INTEGRAL [2] with a flux of $(7.6 \pm 1.5) \times 10^{-12} \mathrm{erg}$ $\mathrm{cm}^{-2} \mathrm{~s}^{-1}$ and $(18.8 \pm 2.8) \times 10^{-12} \mathrm{erg} \mathrm{cm}^{-2} \mathrm{~s}^{-1}$ in $20-40 \mathrm{keV}$ and $40-100 \mathrm{keV}$ bands respectively. It was later observed by XMM-Newton for $\sim 10 \mathrm{ks}$. The broadband analysis of 4C04.42 in $0.2-100$ $\mathrm{keV}$, through non simultaneous XMM-Newton and INTEGRAL data provide strong evidence for soft excess emission below $2 \mathrm{keV}$ (see Figure 3). This excess is well reproduced either with a thermal component with temperature $\mathrm{kT} \sim 0.2 \mathrm{keV}$ or with a broken power-law with $\Delta \Gamma=0.4$ and the break energy $\mathrm{E}_{b r}=2 \mathrm{keV}$.

In Figure 4 we show the SED of $4 \mathrm{C} 04.42$ where non simultaneous data across the electromagnetic spectrum have been taken from NED ${ }^{2}$. In FSRQ the two peaked SED is interpreted in the Synchrotron External Compton framework [14]: the low frequency peak in the IR or FIR is created through the Synchrotron radiation of relativistic electrons moving in a blob, of dimension $\mathrm{R}$, originating in the jet and having a magnetic field of few Gauss. The high frequency peak in the hard-X and gamma-rays is believed to be the effect of IC scattering of the same electrons population on a photon field that can be caused (1) by the Synchrotron radiation (SSC) and/or (2) by thermal emission from the accretion disk, BLR and/or dusty torus (EC). Due to the high density of the external photon field, the EC in FSRQ dominates over the SSC. The secondary effect on the SED derived from EC in the molecular torus, distant on the scale of pc from the central black hole, is not considered here.

A model that well reproduce the $\mathrm{SED}^{3}$ is also included in Figure 4. The simplest hypothesis is that the emitting region is a blob of radius $\mathrm{R}$ moving with a bulk velocity of $\beta \mathrm{c}$ (where $\beta=$

\footnotetext{
${ }^{2}$ NRAO Extragalactic Database; http://nedwww.ipac.caltech.edu/index.html

${ }^{3} \mathrm{SSC}$ code from http://www.asdc.asi.it/ssc_at/. The model was used in e.g. [21] and [6]
} 


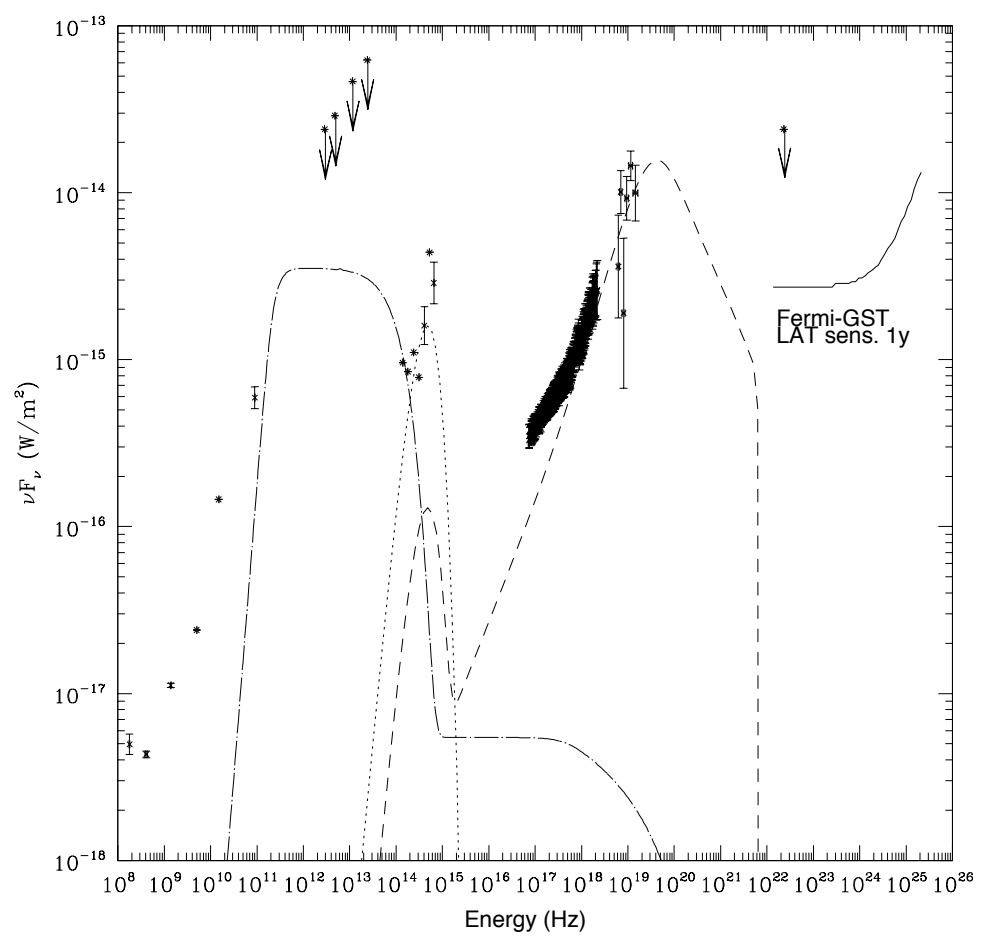

Figure 4: 4C04.42. Spectral Energy Distribution from radio up to soft gamma-ray frequencies. Data are from literature taken by NED. The solid line represents the sensitivity curve for 1 year observation with Fermi-LAT.

$\sqrt{\Gamma^{2}-1} / \Gamma$, and $\Gamma$ is the Lorentz factor), in a magnetic field with intensity of 2 Gauss. We assume an observing angle of $\theta \sim 1 / \Gamma$ implying a Doppler factor $\delta=\Gamma$. The SSC+EC components are plotted separately in Figure 4 with long dashed-dotted and dashed lines respectively. The disk contribution (dotted line) is shown as a thermal component with temperature of $\mathrm{kT}_{B B, z}=1 \mathrm{eV}$ in the quasar rest frame. No other additional component, such as reflection from the disk, has been included in the model. In the figure is also plotted the sensitivity curve for 1 year observation with the Fermi/LAT.

The dimension of the emitting blob, the magnetic field, the electron energy distribution, and the Lorentz factor are the only parameters required to evaluate the SSC component in the SED. We assume that the electrons in the blob are well described by a power-law, $N(\gamma)=\mathrm{N} \gamma^{-p} \mathrm{~cm}^{-3}$, in the range $\gamma_{\min }$ and $\gamma_{\max }$, and we do not take into account radiative cooling of the electrons. We stress that our model for the whole SED is not a true "fit". In view of the not simultaneous data set, we just attempt to extract some physical parameters from the data, with the main goal to obtain average properties of the source. In particular the EC component did allow us to constrain $\gamma_{\text {max }}$, and p. The EC component due to the BLR photon field is determined by the BLR energy density $\mathrm{U}_{B L R}$. We fixed the disk luminosity at the level of the optical-UV emission (at $10^{15} \mathrm{~Hz}$ in the quasar rest frame) observed in the source SED. The BLR luminosity is estimated assuming that the emitting region reprocesses a fraction of the disk luminosity that is $\sim 0.1$ [19]. The energy density in the observer frame is given by $\mathrm{U}_{B L R}^{o s s}=\mathrm{L}_{B L R} / 4 \pi c \mathrm{R}_{B L R}^{2}$, so that the contribution of the IC emission to the 


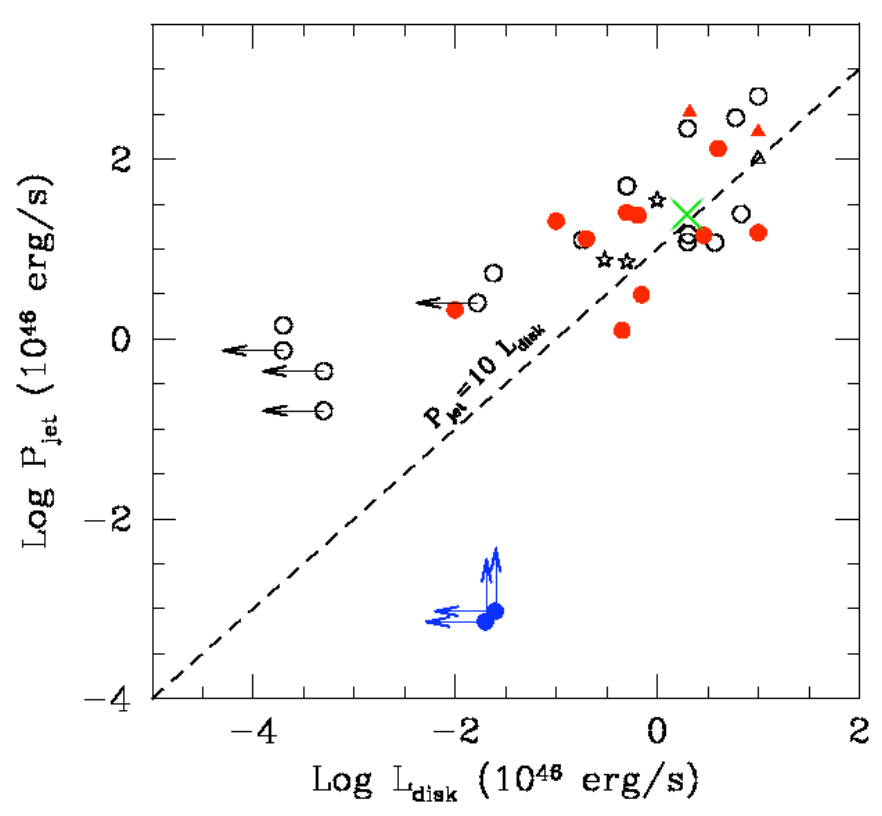

Figure 5: Total jet power vs disk luminosity for different samples of blazars studied in [19, 25, 17]. The green cross represents the location of FSRQ 4C04.42.

SED is $\mathrm{P}_{E C}=4 / 3 \sigma_{T} \mathrm{c} \delta^{4} \int_{\gamma_{\min }}^{\gamma_{\max }} N(\gamma) \gamma^{2} U_{B L R}^{o s s} \Gamma^{2} d \gamma=8 \times 10^{-2} \mathrm{R}_{B L R, 17}^{-2} \mathrm{erg} \mathrm{cm}^{-3} \mathrm{~s}^{-1}$, where $\mathrm{R}_{B L R, 17}$ is the BLR extension in units of $10^{17} \mathrm{~cm} . \mathrm{R}_{B L R}$ is measured in very few AGN through reverberation mapping [15], where a relation between $\mathrm{R}_{B L R}$ and $\lambda \mathrm{L}_{\lambda}(5100 \dot{A})$ is assumed. Taking $\lambda \mathrm{L}_{\lambda}(5100 \dot{A})$ from the $\mathrm{SED}$, we have $\mathrm{R}_{B L R}=5 \times 10^{17} \mathrm{~cm}$. In the emitting volume given by $\mathrm{V}=4 / 3 \pi \mathrm{R}^{3}$, we would then expect $\mathrm{L}_{E C}=3 \times 10^{47} \mathrm{erg} \mathrm{s}^{-1}$, in agreement with the value reported in the SED.

Figure 4 clearly shows evidence that the SSC+EC model does not well fit the whole SED. In particular an excess, of about a factor of three with respect to the EC component, is visible around $2 \mathrm{keV}$. This soft X-ray excess could be due to bulk Compton of a "cold" shell of plasma moving in the jet and interacting with the external BLR photon field [3]. The luminosities of the Bulk and External Compton components, $\mathrm{L}_{B C}$ and $\mathrm{L}_{E C}$, are determined by the relative normalization of the number densities of the cold and relativistic electrons. The number of cold electrons is usually difficult to verify since low energy electrons emit synchrotron light in the self-absorbed range, and SSC radiation at low frequencies, where the flux is dominated by the Synchrotron emission of electrons of higher energies. The only way to observe the bulk Compton component in the SED is when it dominates the non thermal continuum in the soft X-ray energy range, and this will happen when the competitive processes, like SSC, are negligible in this band. However, here we do not try to fit this excess with a bulk Compton component, we just stress that with $\Gamma=20$, as assumed in our model, we expect to detect the peak of such component at the frequency of $\vartheta_{B C}=\Gamma^{2} v_{B L R} /(1+z) \sim$ 
$1 \mathrm{keV}$, i.e. where the excess is indeed observed. In addition, if the excess is interpreted as due to bulk motion then $\mathrm{L}_{B C}=4 / 3 \sigma_{T} \mathrm{c} \delta^{4} \mathrm{U}_{B L R}^{o s s} \Gamma^{2} \mathrm{~N}_{c} \sim 10^{46} \mathrm{~N}_{c} / \mathrm{N}$, where $\mathrm{N}_{c}$ is the number of cold electrons in the blob. With the value we obtained with our broadband analysis, $\mathrm{L}_{B C} \sim 10^{45} \mathrm{erg} \mathrm{s}^{-1}$, we estimate the ratio $\mathrm{N}_{c} / \mathrm{N}$ to be $\sim 0.1$. Very recently the bulk Compton process has been proposed as a means to explain the steepening of the spectrum in the case of quasars PKS 1510-089 at $\mathrm{z}=0.361$ [16], 0723-679 at $\mathrm{z}=0.847,1136-135$ at $\mathrm{z}=0.554$, and $1150-497$ at $\mathrm{z}=0.334$ [25]. The bulk Compton process has been also proposed to explain the observed flattening in the powerful blazar BG B1428+4217, at $z=4.72$ [3]. In this source the soft X-ray behaviour was previously attributed to warm absorption intrinsic to the source $[9,10,27]$. We also stress here that flattening of the spectral emission has been observed in high-z quasars up to $\mathrm{z}=4.4$, while the softening has been observed in very few sources, and all are at redshifts below 1 .

The comparison between the power carried by the jet $\mathrm{P}_{j e t}$, and the disk luminosity $\mathrm{L}_{\text {disk }}$ gives information about important physical quantities Under the assumption of one proton per emitting electron in the jet composition, the jet kinetic power is $\mathrm{P}_{j e t}=\pi \mathrm{R}^{2} \beta \Gamma^{2} \mathrm{cU}$, where $\mathrm{U}=\mathrm{U}_{B}+\mathrm{U}_{e}+\mathrm{U}_{p}$ is the total energy in the jet rest frame due to magnetic field, electron and protons. For 4C04.42, using the model we employed to reproduce the SED and $\gamma_{\text {min }}=1$, we obtain $\mathrm{L}_{\text {disk }} / \mathrm{P}_{\text {jet }} \sim 0.1$ which is in very good agreement with the other cases where this ratio was evaluated with good accuracy $[19,25]$. In the plane $\mathrm{L}_{\text {disk }}$ vs $\mathrm{P}_{j e t}$ (see Figure 5), 4C04.42 lies in the region of high-luminosity blazars, following the trend observed in other similar sources. This, in turn, implies that a large fraction of the accretion power is converted in bulk kinetic energy of the jet $[19,4]$.

\section{IGR J22517-2218: a gamma-ray lighthouse in the Universe}

IGR J22517+2218 was detected by IBIS with a significance of $\sim 7 \sigma$ at a position corresponding to R.A. $(2000)=22 \mathrm{~h} 51 \mathrm{~m} 42.72 \mathrm{~s}$ and $\operatorname{Dec}(2000)=+22^{\circ} 17^{\prime} 56.4^{\prime \prime}$ and with a positional uncertainty of 4.5 ' (90\% confidence level). Follow up observations with the Swift/XRT telescope confirmed the association of IGR J22517+2218 with the high redshift QSO MG3 J225155+2217 at $\mathrm{z}=3.668$. A simple power law model well fit the IBIS data $\left(\chi^{2}=6.5\right.$ for 8 d.o.f.) with a photon index $\Gamma=1.4 \pm 0.6$ combined to an observed $20-100 \mathrm{keV}\left(100-500 \mathrm{keV}\right.$ in the source rest frame) flux of $4 \times 10^{-11} \mathrm{erg}$ $\mathrm{cm}^{-2} \mathrm{~s}^{-1}$.

A power law also provides a good fit to the Swift/XRT data (three available observations) and a trend of decreasing flux from 4.4 to $2.7 \times 10^{-12} \mathrm{erg} \mathrm{cm}^{-2} \mathrm{~s}^{-1}$ becomes evident over a 6 day period. Since the INTEGRAL detection is over a few revolutions, i.e provides an average flux, to improve the statistics the two most statistically significant XRT spectra have been combined. This average spectrum fitted with a power-law has an acceptable $\chi^{2} / \mathrm{dof}=46.9 / 53$ and a flat photon index $(\Gamma=1.31 \pm 0.09)$. However, residuals to this model show some curvature possibly due to intrinsic absorption in the source rest frame (see left panel in Figure 6). Addition of this extra component provides a fit improvement which is significant at the $99.98 \%$ confidence level according to the $\mathrm{F}$ test, a more typical radio-loud AGN spectrum $(\Gamma \sim 1.6)$ and a mild column density $\left(\mathrm{N}_{H}=3 \pm 2 \times\right.$ $10^{22} \mathrm{~cm}^{-2}$ ). However, a broken power law also provides an equally significant improvement in the fit, with a break energy at $1.55 \pm 0.15 \mathrm{keV}$ and a spectral flattening of $\Delta \Gamma=0.5\left(\Gamma_{1}=0.7 \pm 0.3\right.$ and $\Gamma_{2}=1.2 \pm 0.4$ ). While a perfect match in spectral shape with $\Gamma \sim 1.6$ is found, the XRT data fall short 

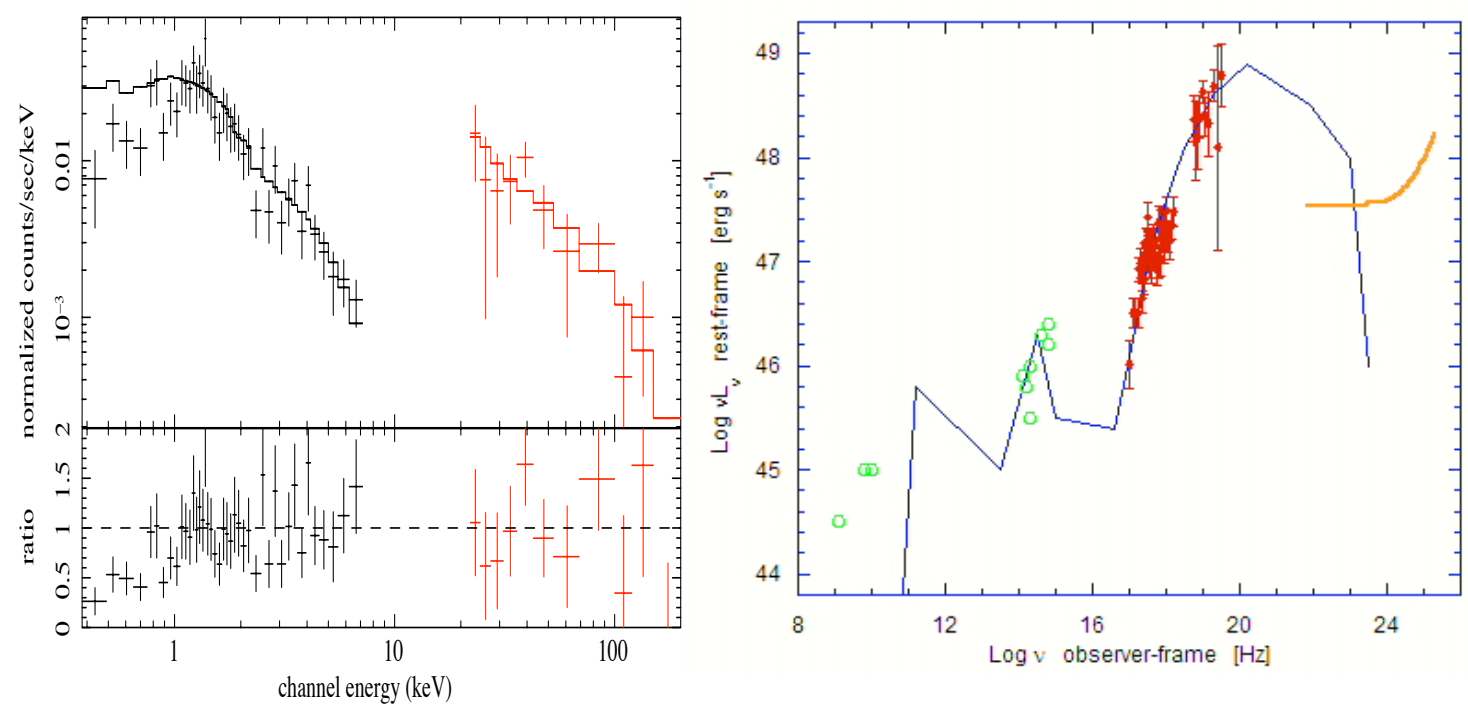

Figure 6: IGR J22517+2218. Left panel: Continuum power-law fit to the energy band above $2 \mathrm{keV}$ (observer frame) extrapolated over the low energy range. Right: Not simultaneous SED from radio frequencies up to gamma-rays. The sensitivity curve for 1 year observation with Fermi-LAT is also shown. The model is that proposed in [17].

of the INTEGRAL detection by a factor in the range 3-7 (see Table 2), implying again variability in the source flux as also evident in the sequence of XRT observations.

The rest frame luminosities are of $0.3 \times 10^{48} \mathrm{erg} \mathrm{s}^{-1}$ in the $\mathrm{X}$-ray $(2-10 \mathrm{keV})$ band, $2 \times 10^{48}$ $\mathrm{erg} \mathrm{s}^{-1}$ at hard X-rays (20-100 keV) and $5 \times 10^{48} \mathrm{erg} \mathrm{s}^{-1}$ in the soft gamma-ray (100-500 KeV) interval, i.e. MG3 J225155+2217 is an X/gamma-ray lighthouse shining from the edge of our Universe.

IGR J2251+2218 is a blazar/FSRQ. The not-simultaneous SED from radio frequencies to gamma-rays is shown in the right panel in Figure 6, the model is that described in [17]. This SED is "unusual" among such type of blazars as it is compatible with having the synchrotron peak in the X/gamma-ray band (i.e. much higher than generally observed) or alternatively with the Compton peak in the $\mathrm{MeV}$ range (i.e. lower than typically measured). Indeed, the $\mathrm{X}$-ray to radio flux ratio is $\sim 1000$ (or $\alpha_{x r}<0.75$ ), similar to high energy peaked blazars, i.e. those with the synchrotron peak in the X-ray band; this is further supported by the observed shape of the infrared to optical continuum which is at odds with the location of the synchrotron peak at infrared frequencies as generally observed in FSRQ. A detailed model of the SED [17] has shown that the hard X-ray INTEGRAL data well define the decreasing part of the EC component. This model has also defined with a good accuracy the location of the EC peak energy, that is around $500 \mathrm{keV}$ observer frame. Either way, MG3 J225155+2217 is quite atypical for its class and an extreme object in the blazar population; this makes it an interesting laboratory in where to test current blazars theories and so an object worth following up at all wavebands. 


\section{Conclusions}

We have presented the broadband spectral study of a small sample of 5 radio-loud QSOs observed whit INTEGRAL, XMM-Newton and Swift. Our main results are:

- All sources but $4 \mathrm{C} 04.42$ are characterized by a $0.2-100 \mathrm{keV}$ spectrum that is best reproduced with an absorbed power-law model. The distribution of $N_{\mathrm{H}}$ is asymmetric, with a peak located at about $\log \mathrm{N}_{\mathrm{H}}=23$, and a tail extending to $\log \mathrm{N}_{\mathrm{H}}=20$. A comparison between the distributions of the column density obtained through the analysis performed in $0.2-10 \mathrm{keV}$ and $0.2-100 \mathrm{keV}$ confirms that the observations above $10 \mathrm{keV}$ represent a strong tool to select heavily obscured objects.

- The mean value of the photon index is lower than that obtained analyzing a sample of QSOs observed in $0.2-10 \mathrm{keV}$ with XMM-Newton. This effect is probably due to the hard X-ray selection that favours the sources with a flatter photon index.

- The broadband analysis of one source of the sample, the FSRQ 4C04.42, shows the presence of a soft excess in the soft X-ray energy range. This excess, as well as the flat spectral shape in hard X -rays, strongly suggests the presence of a population of cold electrons able to produce a bulk Compton feature at $\sim 1 \mathrm{keV}$ through IC with the photon field of the BLR. In addition the ratio $\mathrm{L}_{d i s k} / \mathrm{P}_{\text {jet }} \sim 0.1$ between the disk luminosity and the power carried by the jet, implies that a large fraction of the accretion power is converted in bulk kinetic energy of the jet.

- Among the sample, IGR J22517+2218 is the most distant object so far detected by INTEGRAL and the second most distant blazar ever observed above $20 \mathrm{keV}$. The study of its not-simultaneous SED show evidence that the IC component is dominant with respect to that due to Synchrotron. Observation with Fermi-LAT will be crucial to investigate in depth the emission of this lighthouse in our Universe.

\section{References}

[1] L. Bassani, R. Landi, A. Malizia, M.T. Fiocchi, A. Bazzano, A. J. Bird, A. J. Dean, N. Gehrels, P. Giommi and P. Ubertini, IGR J22517+2218=MG3 J225155+2217: A New Gamma-Ray Lighthouse in the Distant Universe, ApJ 669 (11/2007) L1-L4, [arXiv : 0709 . 3023].

[2] A. J. Bird, A. Malizia, A. Bazzano, E. J. Barlow, L. Bassani, A. B. Hill, G. Belanger, F. Capitanio, D. J. Clark, A. J. Dean, M. Fiocchi, D. Gtz, F. Lebrun, M. Molina, N. Produit, M. Renaud, V. Sguera, J. B. Stephen, R. Terrier, P. Ubertini, R. Walter, C. Winkler and J. Zurita,The Third IBIS/ISGRI Soft Gamma-Ray Survey Catalog, ApJS 170 (05/2007) 175-186.

[3] A. Celotti, G. Ghisellini and A. C. Fabian, Bulk Comptonization spectra in blazars, MNRAS 375 (02/2007) 417-424, [astro-ph / 0611439 ].

[4] A. Celotti and G. Ghisellini, The power of blazar jets, MNRAS 385 (03/2008) 283-300, [arXiv:0711.4112].

[5] A. De Rosa, P. Ubertini, L. Bassani, A. Malizia and A. J. Dean, Bulk Compton motion in the luminous quasar 4C04.42?, MNRAS 388 (07/2008) L54-L58. 
[6] A. De Rosa, L. Piro, A. Tramacere, E. Massaro, R. Walter, L. Bassani, A. Malizia, A. J. Bird and A. J. Dean, The broad-band X-ray spectrum of the blazar PKS B1830-211 by Chandra and INTEGRAL $A \& A 438$ (07/2005) 121-126, [astro-ph/ 0504137 ].

[7] C. D. Dermer and R. Schlickeiser, Model for the High-Energy Emission from Blazars ApJ 416 $(10 / 1993) 458$.

[8] M. Elvis, B. J. Wilkes, J. C. McDowell, C. Jonathan, G. F. Richard, J. Bechtold, S. P. Willner, M. S. Oey, E. Polomski, R. Cutri, Atlas of quasar energy distributions ApJS 95 (11/1994) 1-68.

[9] A. C. Fabian, K. Iwasawa, R. G. McMahon, A. Celotti, W. N. Brandt and M. Hook, The ASCA spectrum of the $Z=4.72$ blazar GB 1428+4217 MNRAS 295 (03/1998) L25-L28, [astro-ph/9801288].

[10] A.C. Fabian, A. Celotti, K. Iwasawa, R. G. McMahon, C. L. Carilli, W. N. Brandt, G. Ghisellini and I. M. Hook, PMN J0525-3343: soft X-ray spectral flattening in a blazar at $z=4.4$ MNRAS 323 (05/2001) 373-379, [astro-ph/0011566].

[11] F. Fiore, M. Elvis, P. Giommi and P. Padovani, X-Ray Spectral Survey of WGACAT Quasars. I. Spectral Evolution and Low-Energy Cutoffs ApJ 492 (01/1998) 79, [astro-ph/9708049].

[12] L. Foschini, G. Tagliaferri, E. Pian, G. Ghisellini, A. Treves, L. Maraschi, F. Tavecchio, G. di Cocco and S. R. Rosen, Simultaneous X-ray and optical observations of S5 0716+714 after the outburst of March 2004 A\&A 455 (09/2006) 871-877, [astro-ph / 0604600 ].

[13] G. Fossati, L. Maraschi, A. Celotti, A. Comastri, G. Ghisellini, A unifying view of the spectral energy distributions of blazers MNRAS 299 (09/1998) 433-448, [astro-ph/9804103].

[14] G. Ghisellini, A. Celotti, G. Fossati, L. Maraschi and A. Comastri, A theoretical unifying scheme for gamma-ray bright blazers MNRAS 301 (12/1998) 451-468, [astro-ph/9807317].

[15] S. Kaspi, D. Maos, H. Netzer, P. M. Bradley, M. Vestergaard and J. T. Buell, The Relationship between Luminosity and Broad-Line Region Size in Active Galactic Nuclei ApJ 629 (08/2005) 61-71, [astro-ph/0504484].

[16] J. Kataoka, G. Madejski, M. Sikora, P. Roming, M. M. Chester, D. Grupe, Y. Tsubuku, R. Sato, N. Kawai, G. Tosti, D. Impiombato, Y. Y. Kovalev, Y. A. Kovalev, P. G. Edwards, S. J. Wagner, R. Moderski, L. Stawarz, T. Takahashi and S. Watanabe, Multiwavelength Observations of the Powerful Gamma-Ray Quasar PKS 1510-089: Clues on the Jet Composition ApJ 672 (01/2008) 787-799, [arXiv: 0709.1528$]$.

[17] L. Maraschi, L. Foschini, G. Ghisellini, F. Tavecchio and R. M. Sambruna, Testing the blazar spectral sequence: X-ray-selected blazers MNRAS 391 (12/2008) 1981-1993, [arXiv : 0810 . 0145].

[18] L. Maraschi, G. Ghisellini and A. Celotti, A jet model for the gamma-ray emitting blazar 3C 279 ApJ 397 (09/1992) L5-L9.

[19] L. Maraschi and F. Tavecchio, The Jet-Disk Connection and Blazar Unification ApJ 593 (08/2003) 667-675, [astro-ph/0205252].

[20] N. Masetti, E. Mason, R. Landi, P. Giommi, L. Bassani, A. Malizia, A. J. Bird, A. Bazzano, A. J. Dean, N. Gehrels, E. Palazzi and P. Ubertini, High-redshift blazar identification for Swift J1656.3-3302 A\&A 480 (03/2008) 715-721, [arXiv : 0801 . 2976].

[21] E. Massaro, A. Tramacere, M. Perri, P. Giommi and G. Tosti, Log-parabolic spectra and particle acceleration in blazars. III. SSC emission in the TeV band from Mkn501 A\&A 448 (03/2006) 861-871, [astro-ph/0511673]. 
[22] K. L. Page, J. N. Reeves, P. T. O'Brien, and M. J. L. Turner, XMM-Newton spectroscopy of high-redshift quasars MNRAS 364 (11/2005) 195-207, [astro-ph/ 0508524 ].

[23] E. Piconcelli, E. Jimenez-Bailn, M. Guainazzi, N. Schartel, P. M. Rodrguez-Pascual and M. Santos-Lle, The XMM-Newton view of $P G$ quasars. I. X-ray continuum and absorption A\&A 432 (03/2005) 15-30, [astro-ph/0411051].

[24] J. N. Reeves and M. J. L. Turner, X-ray spectra of a large sample of quasars with ASCA MNRAS 316 (08/2000) 234-248, [astro-ph/0003080].

[25] R. M. Sambruna, M. Gliozzi, F. Tavecchio, L. Maraschi and L. Foschini, The Jet-Disk Connection in AGNs: Chandra and XMM-Newton Observations of Three Powerful Radio-Loud Quasars ApJ 652 (11/2006) 146, [astro-ph/0606659].

[26] R. M. Sambruna, J. N. Reeves and V. Braito, The Remarkable X-Ray Spectrum of the Broad-Line Radio Galaxy 3C 445 ApJ 665 (08/2007) 1030-1037, [arXiv : 0704 . 3053].

[27] W. Yuan, A. C. Fabian, M. A. Worsley and R. G. McMahon, X-ray spectral properties of high-redshift radio-loud quasars beyond redshift 4 - first results* MNRAS 368 (05/2006) 985-992, [astro-ph/0602272].

[28] S. J. Wagner, M. Camenzind, O. Dreissigacker, U. Borgeest, S. Britzen, W. Brinkmann, U. Hopp, K. J. Schramm and J. von Linde, Simultaneous optical and gamma-ray flaring in PKS 0420-014. Implications for emission processes and rotating jet models A\&A 298 (06/1995) 688. 